\title{
Prevalence of isoniazid resistance-conferring mutations associated with multidrug-resistant tuberculosis in Free State Province, South Africa
}

\author{
L Pitso, ${ }^{1}$ MB ChB, MMed (Internal Medicine); S Potgieter, ${ }^{2}$ MB ChB, MMed (Internal Medicine); A van der Spoel van Dijk, ${ }^{3}$ MSc \\ ${ }^{1}$ Department of Internal Medicine, Faculty of Health Sciences, University of the Free State, Bloemfontein, South Africa \\ ${ }^{2}$ Division of Infectious Diseases, Department of Internal Medicine, Faculty of Health Sciences, University of the Free State, Bloemfontein, South Africa \\ ${ }^{3}$ Department of Medical Microbiology, Faculty of Health Sciences, University of the Free State and National Health Laboratory Service, Bloemfontein, \\ South Africa
}

Corresponding author: L Pitso (pitsol@ufs.ac.za)

Background. Multidrug- and extensively drug-resistant tuberculosis (MDR-TB and XDR-TB) threaten local and global control of the disease. The molecular line-probe assay (LPA) provides rapid diagnosis and early management of MDR-TB. The LPA detects mutations of kat $G$ and inh $A$ genes associated with isoniazid (INH) resistance in Mycobacterium tuberculosis isolates. The kat $G$ and inhA genes are associated with high- and low-level INH resistance, respectively, as well as cross-resistance to ethionamide in the case of inhA gene mutations. Patients with MDR-TB due to an inhA mutation could benefit from the use of high-dose INH - instead of ethionamide - in their MDR-TB regimen.

Objectives. To determine the frequencies of $k a t G$ and inhA mutations that conferred INH resistance among MDR-TB isolates during 2014 - 2016 in Free State (FS) Province of South Africa.

Methods. We retrospectively reviewed MDR-TB isolates assayed with GenoType MTBDRplus (Hain Lifescience, Germany) (LPA) at the central TB laboratory of Universitas Academic Hospital, Bloemfontein, FS, and calculated the frequencies of kat $G$ and inhA mutations. Results. Among 918 MDR-TB isolates, the prevalence of $k a t G$, inh $A$ and $k a t G$ plus $i n h A$ mutations was $63.9 \%, 13.4 \%$ and $22.7 \%$, respectively. Approximately $60 \%$ ( $n=536 ; 58.4 \%)$ of the isolates were obtained from male patients. The patients' ages ranged from 1 to 89 (median 37 ) years. The Xhariep district had the highest incidence of INH resistance-conferring mutations in the province.

Conclusions. kat $G$-associated mutations are the predominant INH resistance-conferring mechanism among MDR-TB isolates in the FS. Patients infected with isolates that harbour the $k a t G$ mutation are unlikely to benefit from high-dose INH therapy in the bedaquiline (BDQ)-containing modified short MDR-TB regimen. They may, however, benefit from the inclusion of ethionamide in the regimen. Dual kat $G$ and $i n h A$ gene mutations make these patients unlikely to respond to either high-dose INH or ethionamide and should now be considered for either the BDQ-containing long MDR-TB regimen or an individualised treatment regimen, depending on fluoroquinolone susceptibility. Clinicians should familiarise themselves with interpreting various INH resistance-conferring mutation results and their implications for management of MDR-TB treatment.

S Afr Med J 2019;109(9):659-664. https://doi.org/10.7196/SAMJ.2019.v109i9.13730

Tuberculosis (TB) is an infectious disease caused by the bacillus Mycobacterium tuberculosis. It remains a major global health challenge and is the leading cause of death related to infection worldwide, exceeding HIV/AIDS. ${ }^{[1]}$

South Africa (SA) is among the top 20 high-burden countries in the world regarding $\mathrm{TB}, \mathrm{TB} / \mathrm{HIV}$ co-infection and multidrugresistant TB (MDR-TB) ${ }^{[1]}$ Worldwide, $\sim 10.4$ million new cases of TB were diagnosed in 2016. Mortality remains high, with $\sim 1.3$ million TB deaths globally in 2016, and an additional 0.37 million deaths resulting from TB disease among HIV-positive individuals. ${ }^{[1]}$

Drug-resistant TB (DR-TB) threatens global TB control measures. A total of 490000 new cases of MDR-TB were reported in 2016. MDR-TB is defined as TB strains with resistance to both isoniazid (INH) and rifampicin. ${ }^{[1,2]}$ Trends among high-burden countries indicate an increase in MDR-TB cases, with the burden of MDR-TB increasing faster or decreasing slower than the overall TB burden. ${ }^{[1]} \mathrm{SA}$ had 19000 MDR-TB cases in 2016. SA also has one of the highest treatment failure rates among the 30 countries with a high burden of MDR-TB, with only 54\% treatment success among patients with MDR-TB and rifampicin-resistant TB (RR-TB) in 2014. ${ }^{[1]}$
Various treatment strategies for the management of MDR-TB are available. The National Department of Health $(\mathrm{NDoH})$ guideline for the management of DR-TB in SA was updated in 2013 and recommends a standardised approach for the management of MDR$\mathrm{TB} .{ }^{[3]}$ A standardised treatment approach relies on the knowledge of country-specific drug-resistance profiles and the frequency with which common second-line agents have been used as part of a programmatic approach in a specific geographical area. ${ }^{[2,3]}$ A programmatic approach is ideally suited to settings where reliable individual drug-susceptibility testing (DST) is not readily available. ${ }^{[2]}$ In SA, the recommended standardised regimen is an intensive phase consisting of 5 different drugs for 6 months. These agents are an injectable aminoglycoside (kanamycin or amikacin), moxifloxacin, ethionamide, terizidone and pyrazinamide (PZA). The aminoglycoside is then discontinued and a continuation phase comprising the remaining 4 drugs for 18 months is recommended. ${ }^{[3]}$ In 2016, the World Health Organization (WHO) issued guidelines on the new shorter MDR-TB drug regimen lasting $<12$ months. This standardised regimen consists of a $4-6$ months' intensive phase with kanamycin, moxifloxacin, prothionamide, clofazimine, PZA, high-dose INH and ethambutol. The continuation 
phase comprises an additional 5 months, consisting of moxifloxacin, clofazimine, PZA and ethambutol. ${ }^{[4]}$

With recent advances in TB diagnostics, molecular testing provides rapid diagnosis and early management of DR-TB. The first-line molecular line-probe assay (LPA) GenoType MTBDRplus (Hain Lifescience, Germany) allows for rapid detection of resistance to rifampicin and INH. ${ }^{[1,2,5]}$ This LPA is based on the extraction and amplification via multiplex polymerase chain reaction (PCR), and subsequent detection of $M$. tuberculosis DNA on a membrane strip using reverse hybridisation ${ }^{[5]}$ It facilitates the detection of the most common mutations in the $r p o B$ gene associated with rifampicin resistance, high-level INH resistance due to mutations in the $k a t G$ gene and low-level INH resistance due to inhA gene mutations. ${ }^{[2,5]}$ LPA was endorsed by the WHO in 2008 for use on smear-positive sputum specimens and clinical isolates of $M$. tuberculosis grown on conventional cultures. ${ }^{[1,2]}$ In 2011, the GenoType MTBDRplus version 2 assay was validated for use in both smear-positive and smear-negative sputum samples. ${ }^{[6,7]}$ The first-line LPA has a laboratory turnaround time of $\sim 5$ hours, allowing for both rapid diagnosis of MDR-TB and early initiation of treatment. ${ }^{[5,8]}$ This test is now readily available in the SA public sector through the National Health Laboratory Service (NHLS).

Mutations resulting in rifampicin resistance are typically located in an 81 base-pair region within the $r p o B$ gene, usually referred to as the rifampicin-resistance determining region (RRDR). More than $95 \%$ of mutations associated with rifampicin resistance occur in this region. ${ }^{[2,9]}$ Although INH resistance is associated with resistance-conferring mutations in multiple genes, $k a t G$ and inhA account for $\sim 90 \%$ of resistance detected by phenotypic DST. $^{[9,10]}$

INH was first reported to be an effective antituberculosis drug in 1952. ${ }^{[11]}$ Mutations conferring INH resistance have since emerged and are associated with deletions of the catalase-peroxidase gene (katG), and/or mutations in the inhA gene responsible for mycolic acid synthesis. The kat $G$ mutations are associated with high-level INH resistance and inhA mutations with low-level resistance. ${ }^{[1]}$ In the presence of inhA mutations, significant cross-resistance between INH and ethionamide has been observed. ${ }^{[2,9]}$ Prior to the widespread use of first-line LPA and routine reporting of specific INH resistanceconferring mutations, ethionamide was considered to be one of the active drugs used as part of standardised MDR-TB treatment in SA. ${ }^{[3]}$ However, use of ethionamide in the presence of a known inh $A$ mutation is regarded as ineffective. In this setting, its use could not only cause unwanted adverse effects without clear benefit to the patient, but could contribute to the development of further resistance by exposing the patient to an inadequate regimen. ${ }^{[5]}$ There is evidence, however, to support the use of high-dose INH in patients with MDRTB with a known inhA mutation. ${ }^{[2,12]}$ Use of LPA has made it possible to rapidly diagnose MDR-TB and provide information regarding the common mutations conferring resistance to both rifampicin and INH. The mutation type associated with INH resistance was not routinely reported by the TB laboratory in the FS until late 2015. Therefore, this information was not previously available to the attending clinician.

The contribution of kat $G$, inh $A$ and $k a t G$ plus inh $A$ mutations in the setting of MDR-TB is not known in the FS. The aim of this study was to retrospectively determine the prevalence of $k a t G, i n h A$ and $k a t G$ plus inh $A$ genetic mutations associated with INH resistance in patients from the FS with MDR-TB identified by LPA during $2014-2016$.

\section{Methods \\ Setting}

In the FS, first-line TB LPA tests are referred to a central TB laboratory at Universitas Academic Hospital, Bloemfontein. The province has a population of $\sim 2.7$ million. ${ }^{[13]}$ It has the fifth highest incidence of TB in SA, with 575 cases per 100000 population. ${ }^{[14]}$ The province had 267 laboratory-confirmed MDR-TB cases in 2010, representing $3.6 \%$ of the national burden. ${ }^{[3]}$ All 5 provincial districts and their respective municipalities refer samples to the central TB laboratory for first-line LPA testing when appropriate, as indicated on a map of the FS showing the different districts and municipalities (Fig. 1). ${ }^{[13,15]}$ On average, the laboratory annually performs 2300 firstline LPA tests.

\section{Data collection}

Data of all LPA tests ( $N=6$ 648) performed from January 2014 to December 2016 were reviewed retrospectively. Smear-positive clinical samples and/or culture-positive isolates across all age and gender groups were included in the final analysis if $M$. tuberculosis complex was detected and resistance to both rifampicin and INH was confirmed. The performance of LPA on smear-negative samples was not validated at the time; these results were excluded from the analysis.

Data were retrieved from LPA reports retained on site at the central TB laboratory in the FS, as well as the corporate data warehouse at the NHLS head office in Johannesburg. The NHLS corporate data warehouse stores laboratory tests and demographic details for all patients receiving healthcare in the public sector, amounting to $~ 80 \%$ of the total SA population. Data were screened for duplication, and where multiple isolates from one patient were encountered, only the first sample was used. Data were sorted according to the type of mutation (kat $G$, inh $A$ or both), age, gender and district municipality.

\section{Analysis}

All data were entered into a Microsoft Excel (Microsoft, USA) workbook for analysis. Descriptive statistics, i.e. frequencies and percentages for categorical data, were calculated. The prevalence

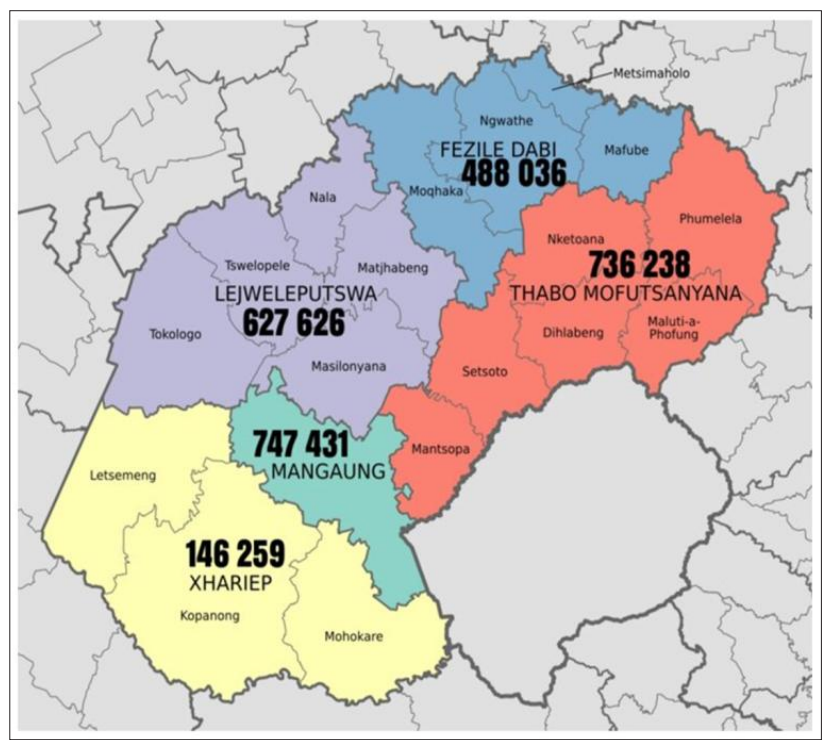

Fig. 1. Map of Free State Province showing one metro (Mangaung), four districts and their municipalities. The numbers represent the population per district. 
of katG, inhA and a combination of the two mutations was calculated for the total samples per year and per district, and described by means of $95 \%$ confidence intervals (CIs). Population data were used to calculate prevalence per 100000 population.

\section{Ethical approval}

This study was approved by the Ethics Committee of the Faculty of Health Sciences, University of the Free State (ref. no. ECUFS 223/2015). Informed consent was not deemed necessary by the committee, as the data included in the study were collected previously during clinical care, and confidential data were accessed only by authorised personnel conducting the study, who signed a confidentiality agreement with the laboratory. To ascertain anonymity, personal information was excluded from the final analysis once data were sorted.

\section{Results}

A total of 6648 LPA test results were reviewed. Of these, 918 (13.8\%) isolates fulfilled the inclusion criteria and were available for the final analysis. The results represented patients from the entire FS population from 2014 to 2016 . The remaining 5730 data results were excluded from the study for several reasons (Fig. 2), including missing patient information, rifampicin or INH mono-resistance, non-tuberculosis mycobacteria, and data duplication due to more than one sample from a single patient submitted during the study period.

The median age of patients whose test results were recorded was 37 (range 1 - 89) years. More than half of the patients were male $(n=536 ; 58.4 \%)$. As shown in Table 1 , of the 918 test results included in the study, 587 (63.9\%) isolates had only kat $G$ mutations detected by LPA, 123 (13.4\%) had only inhA mutations, and the remaining 208 (22.7\%) had both $k a t G$ and inhA mutations. Table 2 summarises the total number of mutations for each year of the study period.

Table 3 shows that the highest number of samples included in the study $(n=312$; $34.0 \%$ ) was received from the Mangaung metropolitan municipality and the lowest number $(n=63 ; 6.9 \%)$ from the Xhariep district. The number of mutations relative to population size was 43/100 000 and 22/100 000 in Xhariep and Fezile Dabi districts, respectively (Table 3).

\section{Discussion}

We examined the local prevalence of the most common mutations of the inhA and kat $G$ genes associated with INH resistance among MDR-TB isolates. To our knowledge, this is the first study in the FS to report findings on this matter.

The $k a t G$ gene mutation, conferring highlevel INH resistance, was found in $63.9 \%$ of MDR-TB isolates tested, which was consistent with previous research identifying $k a t G$ as the predominant gene associated with INH resistance in MDR-TB isolates. An Indian study showed that $94 \%$ of MDR-TB isolates had a $k a t G$ mutation. ${ }^{[16]}$ Barnard et al. ${ }^{[17]}$

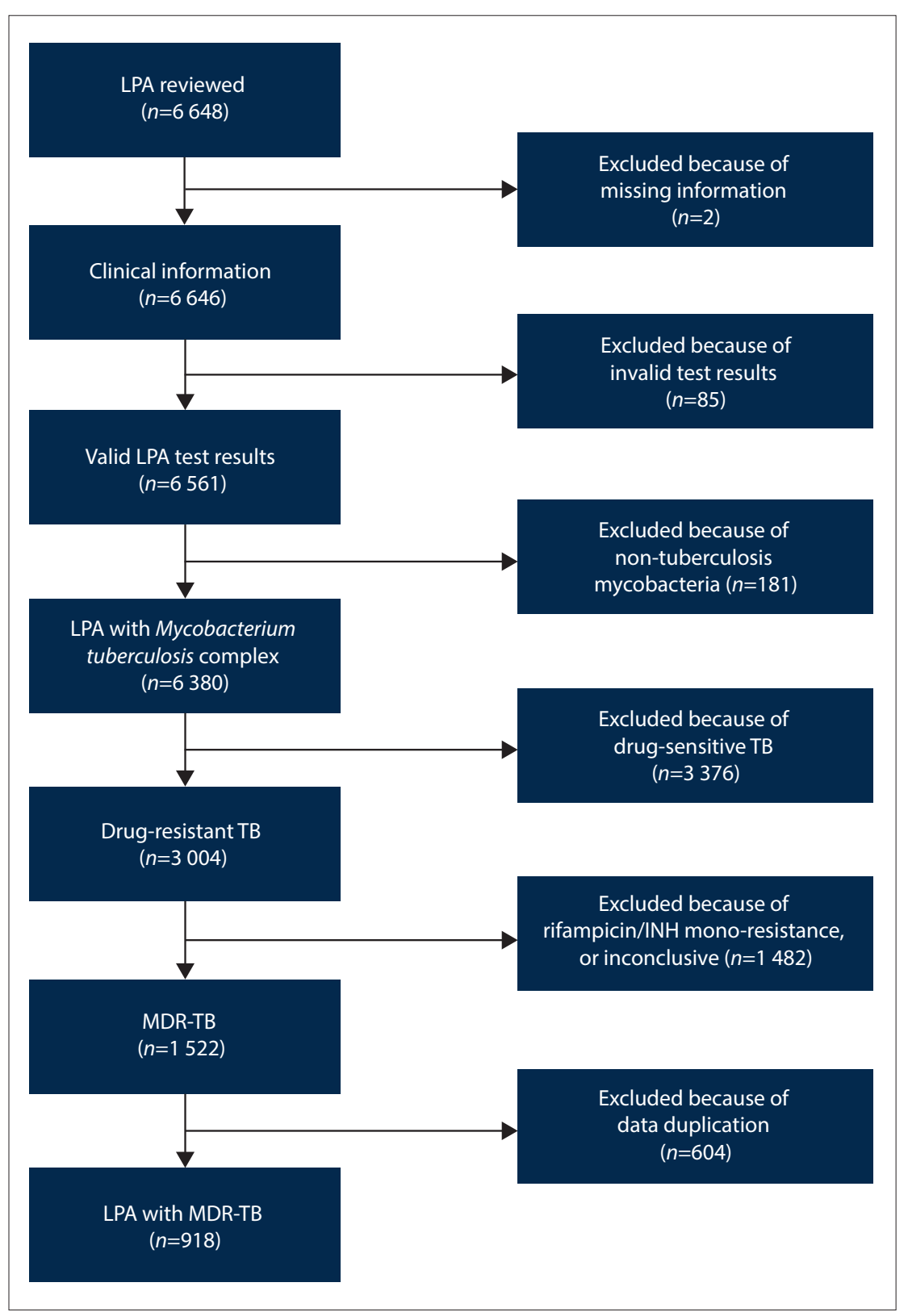

Fig. 2. Diagrammatical summary of data collection and study criteria. ( $L P A=$ line-probe assay; $T B=$ tuberculosis; $I N H=$ isoniazid; $M D R=$ multidrug-resistant.)

Table 1. Frequency $(n)$ and type of mutation in multidrug-resistant isolates of Mycobacterium tuberculosis $(N=918)$

\begin{tabular}{lll}
\hline Gene & $\boldsymbol{n}(\%)$ & $\mathbf{9 5 \%}$ CI (\%) \\
\hline kat $G$ only (high-level INH) & $587(63.9)$ & $60.8-67.0$ \\
inhA only (low-level INH plus ethionamide resistance) & $123(13.4)$ & $11.3-15.8$ \\
kat $G$ and inh $A$ combined & $208(22.7)$ & $20.1-25.5$ \\
CI = confidence interval; INH = isoniazid. & &
\end{tabular}


Table 2. Mutation frequency $(n)$ among multidrug-resistan Mycobacterium tuberculosis isolates by year

Mutation

\begin{tabular}{llll}
\cline { 2 - 4 } Year & & & inhA and $\boldsymbol{k a t} \mathbf{G}$, \\
\hline $2014(n=347)$ & $42(12.1)$ & $213(61.4)$ & $92(26.5)$ \\
$2015(n=267)$ & $37(13.9)$ & $167(62.6)$ & $63(23.6)$ \\
$2016(n=304)$ & $44(14.5)$ & $207(68.1)$ & $53(17.4)$ \\
Total $(N=918)$ & $123(13.4)$ & $587(63.9)$ & $208(22.7)$
\end{tabular}

Table 3. Frequency $(n)$ of isoniazid mutations ${ }^{*}$ among multidrugresistant Mycobacterium tuberculosis isolates $(N=918)$ by district

\begin{tabular}{lll}
\hline District municipality & $\boldsymbol{n}(\%)$ & $\begin{array}{l}\text { Total per } \mathbf{1 0 0 0 0 0} \\
\text { population, } \boldsymbol{n}\end{array}$ \\
\hline Fezile Dabi & $108(11.8)$ & 22 \\
Lejweleputswa & $259(28.2)$ & 41 \\
Mangaung metro & $312(34.0)$ & 42 \\
Thabo Mofutsanyana & $176(19.2)$ & 24 \\
Xhariep & $63(6.9)$ & 43
\end{tabular}

${ }^{*}$ Isoniazid mutations include isolates with either kat $G$, inh $A$ or both $k a t G$ and $i n h A$.

reported a mutation in the $k a t G$ gene in $64.1 \%$ of all INH-resistant strains in the public sector in Western Cape Province of SA, of which $70.8 \%$ were MDR-TB strains. ${ }^{[17]}$

Our finding that $13.4 \%$ of MDR-TB isolates had an inhA gene mutation was comparable with results reported by Yadav et al., ${ }^{[16]}$ who documented an inhA gene mutation in $14 \%$ of MDR isolates. However, the study conducted in the Western Cape showed that $41.9 \%$ (38.2\% of MDR-TB strains) had a mutation in the inhA gene. ${ }^{[17]}$

Dual inhA and kat $G$ mutations were observed among $22.7 \%$ of strains in our study, which were higher than those documented in both the Indian $(6 \%)^{[16]}$ and Western Cape $(10.5 \%)^{[17]}$ studies. It should be noted, however, that the Indian study investigated MDR isolates specifically, ${ }^{[16]}$ while the Western Cape group included all INH-resistant isolates in their study. ${ }^{[17]}$ In KwaZulu-Natal, a province with a high TB prevalence, $15 \%$ of MDR-TB isolates showed dual $k a t G$ and $i n h A$ gene mutations. ${ }^{[18]}$ Several other studies worldwide have shown similar results, but with considerable geographical variation..$^{[9,19-22]}$

While kat $G$ is known to confer high-level resistance to INH with no documented cross-resistance, inh $A$ gene mutations are associated with low-level INH resistance and cross-resistance to ethionamide. . $2,9,11,23]^{2}$ Ethionamide was recommended in the $2013 \mathrm{NDoH}$ guideline on $\mathrm{DR}-\mathrm{TB}$, and was included as part of the standardised treatment regimen for MDR-TB in the SA setting. ${ }^{[3]}$ Consequently, it was commonly used in the management of all MDR-TB patients in the FS until the end of 2015, regardless of the type of INH resistanceconferring mutation. Therefore, up to $36.1 \%$ of patients (those with TB strains harbouring an inhA gene mutation) might have been treated inadequately. The use of ethionamide in the presence of inhA mutations would not have been effective against MDR$\mathrm{TB}$ owing to this cross-resistance. ${ }^{[2]}$ In the absence of the clear benefits of ethionamide, the risk of known adverse effects such as hypothyroidism, teratogenicity, nausea and vomiting may be unacceptably high in this instance. Furthermore, ethionamide is more expensive and not as widely available as INH. ${ }^{[12,24,25]}$

Katiyar et al., ${ }^{[12]}$ in a randomised study, showed that high-dose INH ( $16-18 \mathrm{mg} / \mathrm{kg} /$ day) benefit patients with MDR-TB. They found significant improvement in the time to sputum culture conversion and the likelihood of patients being sputum culture-negative at 6 months compared with placebo and normal-dose INH. ${ }^{[12]}$ Victor et al. ${ }^{[25]}$ also supported the use of high-dose INH, as their study demonstrated that $\sim 50 \%$ of INH-resistant isolates had low minimal inhibitory concentrations (MICs) that could be overcome by using higher doses of INH. ${ }^{[25]}$ Another prospective study of children $<13$ years old conducted in the Western Cape, found that $>80 \%$ of INH monoresistant and MDR infections could be treated with high-dose INH $\left(15-20 \mathrm{mg} / \mathrm{kg} /\right.$ day). ${ }^{[26]}$

A kat $G$ mutation confers high-level INH resistance and an inhA mutation confers ethionamide cross-resistance. ${ }^{[2,9,11,23]}$ Therefore, patients with dual $i n h A$ and $k a t G$ mutations (22.7\% in our study) are unlikely to respond to either high-dose INH or ethionamide, and therefore need to be considered for an individualised TB regimen.

The outcome of MDR-TB treatment has been notoriously poor using conventional 18 - 24-month regimens, with a success rate of $54 \% .{ }^{[1]}$ Not only are these regimens lengthy, posing an adherence problem, but they involve the use of second-line drugs, which are not only less effective, but more expensive and have a worse side-effect profile than first-line drugs. ${ }^{[3]}$ This has led to a search for shorter, more effective regimens. A number of trials have recently proven the effectiveness of a 9 - 12-month regimen. ${ }^{[27-30]}$ In 2016, the WHO endorsed the use of an MDR-TB short course consisting of an initial phase of kanamycin, moxifloxacin, high-dose INH, ethionamide, clofazimine, PZA and ethambutol. ${ }^{[4,31]}$ The original short-course MDR-TB regimen study was conducted in Bangladesh, India, in a setting where LPA for first-line drug-resistance testing was not performed; therefore, the INH resistance-conferring mutations were not known. Nevertheless, the treatment outcome was good. ${ }^{[32]}$ In our study, $36.1 \%$ of patients had TB infection with strains demonstrating an inhA mutation, which would be expected to render resistance to ethionamide, one of the key drugs in the 2016 short-course regimen. While the Bangladesh cohort demonstrated a good outcome despite not knowing the specific INH-resistance mutation, ${ }^{[32]}$ it is our opinion that taking into account its expense and considerable toxicity, we have to question the use of ethionamide in situations where resistance is clearly known. Since release of the WHO recommendations of $2016,{ }^{[4,31,33]}$ the $\mathrm{NDoH}$ has started implementing this short regimen in qualifying patients, unless their isolates demonstrate dual $\mathrm{kat} G$ and inhA mutations that exclude them from the regimen. ${ }^{[34]}$ In our setting, this will expose the $13.4 \%$ of patients with only an inhA mutation to unnecessary adverse effects of ethionamide.

In the latter part of 2018, the WHO released a rapid communication report further modifying the treatment of MDR-TB to a bedaquiline (BDQ)-containing, injectable-free regimen. ${ }^{[35]} \mathrm{BDQ}$ is one of only two new approved TB drugs (delamanid (DLM) being the second) that have been developed in the past 50 years to treat $\mathrm{TB}^{[36]}$ In response, the $\mathrm{NDoH}$ has released interim guidelines on the implementation of new injectable-free regimens in SA. ${ }^{[37]}$ However, most SA patients still receive the previous long regimen of at least 18 months' duration, and some the short regimen that includes both ethionamide and high-dose INH. ${ }^{[37]}$

With the new injectable-free short regimen that includes the use of $\mathrm{BDQ}$, high-dose INH is retained while ethionamide is removed from the regimen, irrespective of $k a t G$ or inh $A$ mutation. ${ }^{[37]}$ Patients in our study whose isolates showed inhA mutations (13.4\%), would benefit from this modified short regimen.

However, although the modified short regimen appears to be promising, the $63.9 \%$ of isolates that showed high-level resistance due to $k a t G$ mutations would theoretically be expected to benefit from ethionamide instead of high-dose INH. In our view, it would 
be worth following up the group of patients with high-dose INH included in their regimen, despite the known high-level resistance to INH due to a $k a t G$ mutation, to determine if treatment outcomes were replicated. The remaining $22.7 \%$ of isolates with dual $k a t G$ and inhA mutations would not be suitable for this modified short regimen and are recommended for the new long regimen, provided that fluoroquinolone sensitivity is confirmed. ${ }^{[37]}$ In contrast to the short modified regimen where high-dose INH is used, irrespective of the presence of either $k a t G$ or $i n h A$ alone, the guideline offers a different recommendation in the long regimen. In the latter regimen, either ethionamide (only kat $G$ ) or high-dose INH (only inhA) may be used, but neither if both mutations are present. This is particularly important where access to new drugs such as BDQ and DLM is uncertain, in children $<12$ years of age due to safety concerns, or shortage of supply. ${ }^{[37]}$

Our study did not investigate the relationship between INH resistance-conferring mutations and history of prior use of INH. Bollela et al. ${ }^{[38]}$ reported a direct link between prior INH exposure for latent or active TB and predominance of kat $G$ mutations. They found that a katG mutation was more frequent among isolates from patients with a history of INH use than in those with no INH exposure $(68.8 \%$ v. $31.2 \%)$. However, this finding did not represent a statistically significant difference. ${ }^{[38]}$ This subject remains unanswered by our study and could be an area for further research.

We also found a comparatively higher number of INH resistanceconferring mutations per population size in the Xhariep district. Although it is geographically the largest of the 5 districts in the province, it is also the least densely populated. ${ }^{[13,15,39]}$ We found that the incidence of INH resistance-causing mutations was 43/100 000 population in the Xhariep district (Table 3), which was the highest in the province. Several factors could explain this finding. The Xhariep district had the highest TB incidence rate of any district in the FS in 2015 at 784/100 000 population, which was well above the national average of $520 / 100000 \cdot{ }^{[40]}$ Furthermore, this finding was associated with the worst TB patient treatment success rate of $72.8 \%$, which was below the provincial and national averages of $80.1 \%$ and $81 \%$, respectively. ${ }^{[4]}$ This implies that a proportion of patients did not complete the treatment for drug-sensitive TB or did not achieve cure. Both these factors would contribute to further development of drug resistance and continual spread among the uninfected population. Moreover, the loss-to-follow-up rate in 2014 among DR-TB patients was the second highest in the province (27.5\%), close to $10 \%$ more than the national average of $17.9 \% \cdot{ }^{[41]}$ Even though some of these patients might have been lost to follow-up due to death, those who stayed alive would have been treated inadequately, and therefore continue to infect the exposed population and to spread resistant strains. Client adherence to treatment is known to be negatively affected by alcohol abuse, unemployment and poor access to quality healthcare. ${ }^{[42]}$ The Xhariep district has an unemployment rate of $27 \%$, with the age group $15-34$ years having a particularly high rate of $33.8 \%{ }^{[13,41]}$ Healthcare resources are also limited, with only 88 professional nurses and 10 medical practitioners providing services in the area in 2016. ${ }^{[41]}$

Our study revealed that the Mangaung metro had the second highest incidence of INH resistance-conferring mutations (42/100 000 population). It has the highest population of all the districts in the province and is one of the wealthiest economically. ${ }^{[40]}$ The high incidence of INH resistance-associated mutations is possibly due to urbanisation and immigration of people from other districts seeking employment and better health services. Lejweleputswa - a prominent mining industry district $^{[39]}$ - had the third highest incidence (41/100 000) of mutations associated with INH resistance. Despite having a population smaller than Thabo Mofutsanyana (a former homeland and largely agricultural district) ${ }^{[13,39]}$ Lejweleputswa district in our study had nearly double the incidence of INH resistance-conferring mutations than that observed in Thabo Mofutsanayana. This is most likely due to mining and its association with a high incidence of TB due to silica dust exposure among mineworkers that impairs macrophage function, leading to a predisposition to $\mathrm{TB}^{[42]}$

\section{Study limitations}

Certain limitations to our study need to be noted. The retrospective design might have introduced selection or information bias. We also did not have comparative phenotypic DST to correlate the performance and agreement between genotypic and phenotypic DST with regard to the diagnosis of MDR-TB in our setting. However, performance of genotypic/molecular DST has been shown to be comparable with that of phenotypic DST and is unlikely to be a major factor. ${ }^{[16,17,43-45]}$ We did not analyse second-line resistance and some of these isolates might have demonstrated resistance to second-line drugs, which would render them XDR-TB rather than MDR-TB isolates. Further research in this regard is recommended.

\section{Conclusions}

We investigated the prevalence of INH resistance-conferring mutations in the FS and found that at least $13.4 \%$ of MDR-TB isolates have an inhA mutation as tested by LPA. Up to $22.7 \%$ of isolates have dual inh $A$ and $k a t G$ gene mutations. The use of ethionamide as part of an MDR-TB regimen in both these groups of patients probably represents an inadequate treatment regimen, with the resultant risk of treatment failure and development of further drug resistance. It also exposes the patient to unnecessary drug toxicity. Likewise, the $63.9 \%$ of patients infected with MDR-TB strains exhibiting high-level INH resistance due to the $k a t G$ mutation, are unlikely to benefit from high-dose INH in the BDQ-containing modified MDR-TB short regimen and may benefit from the use of ethionamide. As these mutations are now routinely reported by the TB laboratory in the FS, it is of vital importance that clinicians know how to interpret the results and are aware of the treatment implications of TB associated with the various INH resistance-conferring mutations. This knowledge of genetic mutations associated with INH resistance and effective implementation of new regimens could help to improve the effectiveness of MDR-TB treatment and thus improve morbidity and reduce further transmission. This needs careful consideration, as new guidelines for the management of MDR-TB are being formulated in SA.

Declaration. The research reported in this article was a requirement for the MMed (Internal Medicine) degree conferred to LP.

Acknowledgements. Ms Riette Nel, Department of Biostatistics, University of the Free State (UFS), for assistance with the analysis of data; Dr Daleen Struwig, medical writer/editor, Faculty of Health Sciences, UFS, for technical and editorial preparation of the manuscript.

Author contributions. LP developed the protocol, collected and interpreted the data and wrote the article; SP and AvdSvD assisted with protocol development, interpretation of data, and writing and editing of the document; all the authors approved the final version of the document. Funding. None.

Conflicts of interest. None.

1. World Health Organization. Global Tuberculosis Report 2017. Geneva: WHO, 2017 2. World Health Organization. Companion Handbook to the WHO Guidelines for the Programmatic Management of Drug-resistant Tuberculosis. Geneva: WHO, 2014.

3. National Department of Health. Management of drug-resistant tuberculosis: Policy guidelines. https: www.health-e.org.za/wp-content/uploads/2014/06/MDR-TB-Clinical-Guidelines-Updated-Jan-2013. pdf (accessed 1 August 2018). 
4. World Health Organization. The shorter MDR-TB regimen. http://www.who.int/tb/Short_MDR_regimen_ factsheet.pdf (accessed 1 August 2018).

5. Hain Lifescience. Rapid diagnostics of tuberculosis and its resistances. http://www.hain-lifescience.de/ Hain Lifescience. Rapid diagnostics of tuberculosis and its resistances. http://www.hain-lifescience
uploadfiles/file/produkte/mikrobiologie/mykobakterien/tb_eng.pdf (accessed 1 August 2018).

6. Seifert M, Ajbani K, Georghiou SB, et al. A performance evaluation of MTBDRplus version 2 for the diagnosis of multidrug-resistant tuberculosis. Int J Tuberc Lung Dis 2016;20(5):631-637. https://doi. org/10.5588/ijtld.15.0788

7. Nathavitharana RR, Hillemann D, Schumacher SG, et al. Multicenter noninferiority evaluation of Hain GenoType MTBDRplus version 2 and Nipro NTM+MDRTB line probe assays for detection of rifampin and isoniazid resistance. J Clin Microbiol 2016;54(6):1624-1630. https://doi.org/10.1128/ JCM.00251-16

8. Tomasicchio M, Theron G, Pietersen E, et al. The diagnostic accuracy of the MTBDRplus and MTBDRsl assays for drug-resistant TB detection when performed on sputum and culture isolates. Sci Rep 2016;6(1):17850. https://doi.org/10.1038/srep17850

9. Zhang Y, Yew WW. Mechanisms of drug resistance in Mycobacterium tuberculosis: Update 2015. Int J Tuberc Lung Dis 2015;19(11):1276-1289. https://doi.org/10.5588/ijtld.15.0389

10. World Health Organization. Tuberculosis diagnostics: Molecular Line-probe Assay for the Detection of Resistance to Isoniazid and Rifampicin (LPA). Geneva: WHO, 2016.

11. Banerjee A, Dubnau E, Quemard A, et al. $\operatorname{inh} A$, a gene encoding a target for isoniazid and ethionamide in Mycobacterium tuberculosis. Science 1994;263(5144):227-230. https:// 1 oi.org/10.1126
etronger science. 8284673

12. Katiyar SK, Bihari S, Prakash S, Mamtani M, Kulkarni H. A randomised controlled trial of high-dose isoniazid adjuvant therapy for multidrug-resistant tuberculosis. Int J Tuberc Lung Dis 2008;12(2):139-145.

13. Statistics South Africa. Census 2011 Municipal Report Free State. Report No. 03-01-52. Pretoria: StatsSA, 2012

4. TBfacts.org. Information about tuberculosis: TB statistics for South Africa - national and provincial. https://www.tbfacts.org/tb-statistics-south-africa/ (accessed 1 August 2018).

15. Wikipedia. List of municipalities in the Free State. https://en.wikipedia.org/wiki/List_of_municipalities_ in_the_Free_State (accessed 1 August 2018).

16. Yadav RN, Singh BK, Sharma SK, et al. Comparative evaluation of GenoType MTBDRplus line probe assay with solid culture method in early diagnosis of multidrug resistant tuberculosis (MDRTB) at a tertiary care centre in India. PLOS ONE 2013:8(9)·e72036. https.//doiorg/10.1371/journal. pone. 0072036

17. Barnard M, Albert H, Coetzee G, OoBrien R, Bosman ME. Rapid molecular screening for multidrug resistant tuberculosis in a high-volume public health laboratory in South Africa. Am J Respir Crit Care Med resistant tuberculosis in a high-volume public health laboratory in S
2008;177(7):787-792. https://doi.org/10.1164/rccm.200709-1436OC

18. Niehaus AJ, Mlisana K, Gandhi NR, Mathema B, Brust JC. High prevalence of $i n h A$ promoter mutations among patients with drug-resistant tuberculosis in KwaZulu-Natal, South Africa. PLOS ONE mutations among patients with drug-resistant tuberculosis in KwaZul
2015;10(9):e0135003. https://doi.org/10.1371/journal.pone. 0135003

19. Luo T, Zhao M, Li X, et al. Selection of mutations to detect multidrug-resistant Mycobacterium tuberculosis strains in Shanghai, China. Antimicrob Agents Chemother 2010;54(3):1075-1081. https:/ doi.org/10.1128/AAC.00964-09

20. Jagielski T, Bakuła Z, Roeske K, et al. Detection of mutations associated with isoniazid resistance in multidrug-resistant Mycobacterium tuberculosis clinical isolates. J Antimicrob Chemother 2014;69(9):2369-2375. https://doi.org/10.1093/jac/dkul61

21. Maurya AK, Singh AK, Kant S, et al. Use of GenoType' MTBDRplus assay to assess drug resistance and mutation patterns of multidrug-resistant tuberculosis isolates in northern India. Indian J Med Microbio 2013;31(3):230-236

22. Kiepiela P, Bishop KS, Smith AN, Roux L, York DF. Genomic mutations in the katG, inhA and aphC genes are useful for the prediction of isoniazid resistance in Mycobacterium tuberculosis isolates from Kwazulu Natal, South Africa. Tuber Lung Dis 2000;80(1):47-56. https://doi.org/10.1054/tuld.1999.0231

23. Partners in Health. The PIH Guide to the Medical Management of Multidrug-resistant Tuberculosis. 2nd ed. Washington, DC: US Agency for International Development, 2013.

24. Resch SC, Salomon JA, Murray M, Weinstein MC. Cost-effectiveness of treating multidrug-resistant tuberculosis. PLoS Med 2006;3(7):e241. https://doi.org/10.1371/journal.pmed.0030241

25. Victor TC, Warren R, Butt JL, et al. Genome and MIC stability in Mycobacterium tuberculosis an indications for continuation of use of isoniazid in multidrug-resistant tuberculosis. J Med Microbiol 1997;46(10):847-857. https://doi.org/10.1099/00222615-46-10-847
26. Schaaf HS, Victor TC, Engelke E, et al. Minimal inhibitory concentration of isoniazid in isoniazidresistant Mycobacterium tuberculosis isolates from children. Eur J Clin Microbiol Infect Dis 2007;26(3):203-205. https:///doi.org/10.1007/s10096-007-0257-9

27. Van Deun A, Maug AK, Salim MA, et al. Short, highly effective, and inexpensive standardized treatment of multidrug-resistant tuberculosis. Am J Respir Crit Care Med 2010;182(5):684-692. treatment of multidrug-resistant tuberculosis.

28. Kuaban C, Noeske J, Rieder HL, Ait-Khaled N, Abena Foe JL, Trébucq A. High effectiveness of a 12-month regimen for MDR-TB patients in Cameroon. Int J Tuberc Lung Dis 2015;19(5):517-524. https://doi.org/10.5588/ijtld.14.0535

29. Piubello A, Harouna SH, Souleymane MB, et al. High cure rate with standardised shortcourse multidrug-resistant tuberculosis treatment in Niger: No relapses. Int J Tuberc Lung Dis 2014;18(10):1188-1194. https://doi.org/10.5588/ijtld.13.0075

30. Nunn AJ, Rusen ID, van Deun A, et al. Evaluation of a standardized treatment regimen of antituberculosis drugs for patients with multidrug-resistant tuberculosis (STREAM): Study protocol for a tuberculosis drugs for patients with multidrug-resistant tuberculosis (STREAM): Study protocol
randomized controlled trial. Trials 2014;15(1):353. http: //doi.org/10.1186/1745-6215-15-353

31. Falzon D, Schünemann HJ, Harausz E, et al. World Health Organization treatment guidelines for drug-resistant tuberculosis, 2016 update. Eur Respir J 2017;49(3):1602308. https://doi.org/10.1183/ 13993003.02308-2016

32. Aung KJ, van Deun A, Declercq E, et al. Successful '9-month Bangladesh regimen' for multidrugresistant tuberculosis among over 500 consecutive patients. Int J Tuberc Lung Dis 2014;18(10):11801187. https://doi.org/10.5588/ijtld.14.0100

33. World Health Organization. WHO Treatment Guidelines for Drug-resistant Tuberculosis. Geneva: WHO, 2016.

34. Ismail NA, Ndjeka N. Introduction of a new rapid diagnostic tool and short-course regimen for drugresistant tuberculosis in South Africa. Public Health Surveill Bull 2017;15(3):96-101.

35. World Health Organization. Rapid Communication: Key Changes to Treatment of Multidrug- and Rifampicin-resistant Tuberculosis (MDR/RR-TB). Geneva: WHO, 2018.

36. National Department of Health. Introduction of new drugs and drug regimens for the management of drug-resistant tuberculosis in South Africa: Policy framework http//wwwtonlineinfo/medint uploads/documents/policy_framework_ver_20150608.pdf (accessed 12 January 2019).

37. National Department of Health. Interim clinical guidance for the implementation of injectablefree regimens for rifampicin-resistant tuberculosis in adults, adolescents and children. http://www. tbonline.info/media/uploads/documents/dr_tb_clinical_guidelines_for_rsa_september_2018.pdf (accessed 12 January 2018).

8. Bollela VR, Namburete EI, Feliciano CS, Macheque D, Harrison LH, Caminero JA. Detection of katG and inhA mutations to guide isoniazid and ethionamide use for drug-resistant tuberculosis. Int $J$ and inhA mutations to guide isoniazid and ethionamide use for drug-resi

39. Main O, ed. The Local Government Handbook South Africa 2018. A Complete Guide to Municipalities in South Africa. 8th ed. Cape Town: Yes Media, 2018.

40. Massyn N, Peer N, English R, Padarath A, Barron P, Day C, eds. District Health Barometer 2015/16. Durban: Health Systems Trust, 2016

41. Massyn N, Padarath A, Peer N, Day C, eds. District Health Barometer 2016/17. Durban: Health Systems Trust, 2017

42. National Department of Health. National tuberculosis management guidelines 2014. http://www.health. gov.za/index.php/shortcodes/2015-03-29-10-42-47/2015-04-30-08-18-10/2015-04-30-08-23-21 (accessed 2 August 2018)

43. Bedewi Omer Z, Mekonnen Y, Worku A, et al. Evaluation of the GenoType MTBDRplus assay for detection of rifampicin- and isoniazid-resistant Mycobacterium tuberculosis isolates in central Ethiopia. Int J Mycobacteriol 2016;5(4):475-481. https://doi.org/10.1016/ji.imyco.2016.06.005

44. Abanda NN, Djieugoué JY, Lim E, et al. Diagnostic accuracy and usefulness of the Genotype MTBDRplus assay in diagnosing multidrug-resistant tuberculosis in Cameroon? A cross-section tudy. BMC Infect Dis 2017;17(1):379. https://doi.org/10.1186/s12879-017-2489-3

45. Aung WW, Ei PW, Nyunt WW, et al. Phenotypic and genotypic analysis of anti-tuberculosis drug resistance in Mycobacterium tuberculosis isolates in Myanmar. Ann Lab Med 2015;35(5):494-499. https://doi.org/10.3343/alm.2015.35.5.494

Accepted 18 March 2019 